Journal of Mathematics and Statistics 3 (4): 201-206, 2007

ISSN 1549-3644

C 2007 Science Publications

\title{
A New Approach to Solve Nonlinear Partial Differential Equations
}

\author{
${ }^{1}$ Abdoul R. Ghotbi, ${ }^{1}$ M. A. Mohammadzade, ${ }^{1}$ A. Avaei and ${ }^{2}$ M. Keyvanipoor \\ ${ }^{1}$ Department of Civil Engineering, Shahid Bahonar University, 22 Bahman Blvd, Kerman, Kerman, Iran \\ ${ }^{2}$ Azad University of Torbate jam, Mashhad, Torbate jam, Iran
}

\begin{abstract}
Nonlinear phenomena play a crucial role in applied mathematics and physics. Explicit solutions to the nonlinear equations are of fundamental importance. Various methods for obtaining explicit solution to nonlinear evolution equations have been proposed. In this letter homotopy perturbation method (HPM) is employed for solving one-dimensional non-homogeneous parabolic partial differential equation with a variable coefficient and a system of nonlinear partial differential equations. The final results obtained by means of HPM, were compared with those results obtained from the exact solution and the Adomian Decomposition Method (ADM). The comparison shows a precise agreement between the results, and introduces this new method as an applicable one which it needs less computations and is much easier and more convenient than others, so it can be widely used in engineering too.
\end{abstract}

Keywords: One-dimensional non-homogeneous parabolic partial differential equation; system of nonlinear equations; Homotopy Perturbation method (HPM).

\section{INTRODUCTION}

Nonlinear phenomena play a crucial role in applied mathematics and physics. Explicit solutions to the nonlinear equations are of fundamental importance. Various methods for obtaining explicit solution to nonlinear evolution equations have been proposed. Hirota's dependent variable transformation, the inverse scattering transform, and the Bäcklund transformation are the most important ones. A common feature to all of these methods is that they are using the transformations to reduce the equation under study into simpler one, and then solve it. Unlike classical techniques, the nonlinear equations are solved easily and elegantly with out transforming or linearizing the equation by using the homotopy perturbation method (HPM). It provides an efficient explicit solution with high accuracy, minimal calculations, and avoidance of physically unrealistic assumptions.

The HPM was first proposed by $\mathrm{He}$, and has been shown to solve a large class of nonlinear problems effectively, easily, and accurately with approximations converging rapidly to accurate solutions. The HPM was proposed to search for limit cycles or bifurcation curves of nonlinear equations.

In a heuristic example was given to illustrate the basic idea of the HPM and its advantages over the $\delta$ - method, the method was also applied to solve boundary value problems, and heat radiation equations ${ }^{[1,2,3-7]}$.

In this study, we implemented the HPM for finding the approximate solutions of one-dimensional nonhomogeneous parabolic partial differential equation with a variable coefficient and a system of nonlinear partial differential equations ${ }^{[8,9]}$.

These two equations are as fallows:

$$
\begin{aligned}
& u_{t}=u_{x x}+\phi(x, t)=u_{x x}+\exp (-x)(\cos t-\sin t), \\
& \text { ' } \mathrm{q}
\end{aligned}
$$

Where the initial condition is $u(x, 0)=f(x)=x$ and the boundary conditions are:

$$
u(0, t)=\sin t, \quad u(1, t)=1+\frac{\sin t}{e} .
$$

Which it is easily can be seen that it has an exact solution of $u(x, t)=x+\exp (-x) \sin t$ [8].

For the second equation, let's consider the system of nonlinear partial differential equations ${ }^{[9]}$ :

$$
\left\{\begin{array}{l}
u_{t}=u u_{x}+v u_{y} \\
v_{t}=u v_{x}+v v_{y}
\end{array}\right.
$$

With the initial conditions

Corresponding Author: A.Ghotbi, Faculty of civil engineering, Shahid Bahonar University, 22 Bahman Blvd, Kerman, Iran. Tel/Fax: +98(21)-33939450. 


$$
\left\{\begin{array}{l}
u(x, y, 0)=x^{2}, \\
v(x, y, 0)=y .
\end{array}\right.
$$

\section{MATERIALS AND METHODS}

Homotopy-perturbation method: To explain this method, let us consider the following function:

$A(u)-f(r)=0, \quad r \in \Omega$

With the boundary conditions of

$$
B\left(u, \frac{\partial u}{\partial n}\right)=0, \quad r \in \Gamma
$$

Where $A, B, f(r)$ and $\Gamma$ are a general differential operator, a boundary operator, a known analytical function and the boundary of the $\operatorname{domain} \Omega$, respectively.

Generally speaking, the operator $A$ can be divided in to a linear part $L$ and a nonlinear part $N$. Eq. (5) can therefore, be written as:

$$
L(u)+N(u)-f(r)=0 .
$$

By the homotopy technique, we construct a homotopy $v(r, p): \Omega \times[0,1] \rightarrow R$ which satisfies:

$$
\begin{aligned}
& H(v, p)=(1-p)\left[L(v)-L\left(u_{0}\right)\right]+ \\
& p[A(v)-f(r)]=0, \\
& p \in[0,1], r \in \Omega, \\
& \text { Or } \\
& H(v, p)=L(v)-L\left(u_{0}\right)+p L\left(u_{0}\right)+ \\
& p[N(v)-f(r)]=0,
\end{aligned}
$$

Where $p \in[0,1]$ is an embedding parameter, while $u_{0}$ is an initial approximation of Eq. (5), which satisfies the boundary conditions. Obviously, from Eqs. (8) and (9) we will have:

$$
\begin{aligned}
& H(v, 0)=L(v)-L\left(u_{0}\right)=0, \\
& H(v, 1)=A(v)-f(r)=0 .
\end{aligned}
$$

The changing process of $p$ from zero to unity is just that of $v(r, p)$ from $u_{0}$ to $u(r)$.In topology, this is called deformation, while $L(v)-L\left(u_{0}\right)$ and $A(v)-f(r)$ are called homotopy.

According to the HPM, we can first use the embedding parameter $p$ as a "small parameter", and assume that the solutions of Eqs. (8) and (9) can be written as a power series in $p$ :

$v=v_{0}+p v_{1}+p^{2} v_{2}+\ldots$

Setting $p=1$ results in the approximate solution of Eq.

(5):

$u=\lim _{p \rightarrow 1} v=v_{0}+v_{1}+v_{2}+\ldots$.

The combination of the perturbation method and the homotopy method is called the HPM, which eliminates the drawbacks of the traditional perturbation methods while keeping all its advantages. The series (13) is convergent for most cases. However, the convergent rate depends on the nonlinear operator $A(v)$. Moreover, the following suggestions were made by He:

(1) The second derivative of $N(v)$ with respect to $\mathrm{v}$ must be small because the parameter may be relatively large, i.e. $p \rightarrow 1$.

(2) The norm of $L^{-1} \frac{\partial N}{\partial v}$ must be smaller than one so that the series converges ${ }^{[1,2,3-7]}$.

Analysis the method: To investigate equation. (1), we first construct a homotopy by separating the linear and nonlinear parts of the equation; we apply homotopyperturbation to Eq. (1) using Eq. (8) as fallows:

$$
\begin{aligned}
& H(v, p)=(1-p)\left(\frac{\partial v(x, t)}{\partial t}-\frac{\partial v_{0}(x, t)}{\partial t}\right)+ \\
& p\left(\frac{\partial v(x, t)}{\partial t}-\frac{\partial^{2} v(x, t)}{\partial^{2} x}-\right. \\
& \left(1-x+0.5 x^{2}\right)\left(1-0.5 t^{2}-t\right)=0
\end{aligned}
$$

Substituting Eq. (12) in to Eq. (14) and rearranging the resultant equation based on powers of $\mathrm{p}$-terms, one has:

$p^{0}: \frac{\partial}{\partial t} v_{0}(x, t)=0$

$p^{1}: \frac{\partial}{\partial t} v_{1}(x, t)-1-\frac{\partial^{2}}{\partial x^{2}} v_{0}(x, t)+$

$t+x-0.5 x t^{2}+0.5 t^{2}-0.5 x^{2}+$

$0.25 x^{2} t^{2}+0.5 x^{2} t-x t=0$,

$p^{2}:-\frac{\partial^{2}}{\partial x^{2}} v_{1}(x, t)+\frac{\partial}{\partial t} v_{2}(x, t)=0$ 
With the following conditions:

$\left\{\begin{array}{l}v_{0}(x, 0)=x, v_{0}(0, t)=\sin (t), v_{0}(1, t)=1+\frac{\sin (t)}{e}, \\ v_{i}(x, 0)=0, v_{i}(0, t)=v_{i}(1, t)=0, i=1,2, \ldots \ldots\end{array}\right.$

In order to obtain the unknowns, we should solve Eqs.

(16) and (17), considering the initial conditions of Eqs.

(18), and having the initial approximations of Eq. (12).

So we have:

$v_{0}(x, t)=x$

$v_{1}(x, t)=-\frac{1}{4}\left(-2 x+2+x^{2}\right)\left(\frac{1}{3} t^{3}+t^{2}\right)+$

$\left(1-x+\frac{1}{2} x^{2}\right) t$

$v_{2}(x, t)=-\frac{1.666666667}{4 \times 10^{10}} t^{4}-\frac{1}{6} t^{3}+\frac{1}{2} t^{2}$

In the same manner, the rest of components were obtained using the maple package.

According to the HPM, we can conclude that

$u(x, t)=\lim _{p \rightarrow 1} v(x, t)=v_{0}(x, t)+v_{1}(x, t)+$

$v_{2}(x, t)+\ldots .$.

Therefore, substituting the values of $v_{0}(x, t), v_{1}(x, t)$ and $v_{2}(x, t)$ from Eqs. (19), (20) and (21) in to Eq. (22) yields:

$$
\begin{aligned}
& u(x, t)=x-\frac{1}{4}\left(-2 x+2+x^{2}\right)\left(\frac{1}{3} t^{3}+t^{2}\right)+ \\
& \left(1-x+\frac{1}{2} x^{2}\right) t-\frac{1.666666667}{4 \times 10^{10}} t^{4}-\frac{1}{6} t^{3}+\frac{1}{2} t^{2}
\end{aligned}
$$

The comparison of the results of HPM and exact solution are illustrated in figs. 1, 2 and 3 respectively.

To investigate Eqs. (3) we first construct a homotopy as fallows: $(1-p)\left[\frac{\partial v_{1}(x, y, t)}{\partial t}\right]+$
$p\left[\begin{array}{l}\left(\frac{\partial v_{1}(x, y, t)}{\partial t}\right)-v_{1}(x, y, t) \frac{\partial v_{1}(x, y, t)}{\partial x}- \\ v_{2}(x, y, t)\left(\frac{\partial v_{1}(x, y, t)}{\partial y}\right)\end{array}\right]=0$

$(1-p)\left[\frac{\partial v_{2}(x, y, t)}{\partial t}\right]+$

$p\left[\begin{array}{l}\left(\frac{\partial v_{2}(x, y, t)}{\partial t}\right)-v_{1}(x, y, t) \frac{\partial v_{2}(x, y, t)}{\partial x}- \\ v_{2}(x, y, t)\left(\frac{\partial v_{2}(x, y, t)}{\partial y}\right)\end{array}\right]=0$

And the initial approximations are as fallows:

$\left\{\begin{array}{l}v_{1,0}(x, y, t)=v_{1}(x, y, 0)=u_{0}(x, y, t)=x^{2} \\ v_{2,0}(x, y, t)=v_{2}(x, y, 0)=v_{0}(x, y, t)=y \\ v_{1, i}(x, y, 0)=v_{2, i}(x, y, 0)=0, \\ i=1,2,3, \ldots .\end{array}\right.$

And

$\left\{\begin{array}{l}v_{1}(x, y, t)=v_{1,0}(x, y, t)+p v_{1,1}(x, y, t)+ \\ p^{2} v_{1,2}(x, y, t)+p^{3} v_{1,3}(x, y, t)+\ldots . . \\ v_{2}(x, y, t)=v_{2,0}(x, y, t)+p v_{2,1}(x, y, t)+ \\ p^{2} v_{2,2}(x, y, t)+p^{3} v_{2,3}(x, y, t)+\ldots . .\end{array}\right.$

Substituting Eqs. (27) in to Eqs. (24) and (25) and arranging the coefficients of $p$ powers, we have

$p^{0}: \frac{\partial v_{1,0}(x, y, t)}{\partial t}=0$

$p^{1}: \frac{\partial v_{1,1}(x, y, t)}{\partial t}-v_{1,0}(x, y, t) \frac{\partial v_{1,0}(x, y, t)}{\partial x}-$

$v_{2,0}(x, y, t) \frac{\partial v_{1,0}(x, y, t)}{\partial y}=0$ 


$$
\begin{aligned}
& p^{2}: \frac{\partial v_{1,2}(x, y, t)}{\partial t}-v_{1,0}(x, y, t) \frac{\partial v_{1,1}(x, y, t)}{\partial x}- \\
& v_{1,1}(x, y, t) \frac{\partial v_{1,0}(x, y, t)}{\partial x}- \\
& v_{2,0}(x, y, t) \frac{\partial v_{1,1}(x, y, t)}{\partial y}- \\
& v_{2,1}(x, y, t) \frac{\partial v_{1,0}(x, y, t)}{\partial y}=0, \\
& p^{3}: \frac{\partial v_{1,3}(x, y, t)}{\partial t}-v_{1,0}(x, y, t) \frac{\partial v_{1,2}(x, y, t)}{\partial x}- \\
& v_{1,1}(x, y, t) \frac{\partial v_{1,1}(x, y, t)}{\partial x}- \\
& v_{1,2}(x, y, t) \frac{\partial v_{1,0}(x, y, t)}{\partial x}- \\
& v_{2,0}(x, y, t) \frac{\partial v_{1,2}(x, y, t)}{\partial y}- \\
& v_{2,1}(x, y, t) \frac{\partial v_{1,1}(x, y, t)}{\partial y}- \\
& v_{2,2}(x, y, t) \frac{\partial v_{1,0}(x, y, t)}{\partial y}=0 .
\end{aligned}
$$

And

$$
p^{0}: \frac{\partial v_{2,0}(x, y, t)}{\partial t}=0
$$$$
p^{1}: \frac{\partial v_{2,1}(x, y, t)}{\partial t}-v_{2,0}(x, y, t) \frac{\partial v_{2,0}(x, y, t)}{\partial y}-
$$$$
v_{1,0}(x, y, t) \frac{\partial v_{2,0}(x, y, t)}{\partial x}=0,
$$$$
p^{2}: \frac{\partial v_{2,2}(x, y, t)}{\partial t}-v_{1,1}(x, y, t) \frac{\partial v_{2,0}(x, y, t)}{\partial x}-
$$$$
v_{2,1}(x, y, t) \frac{\partial v_{2,0}(x, y, t)}{\partial y}-
$$$$
v_{1,0}(x, y, t) \frac{\partial v_{2,1}(x, y, t)}{\partial x}-
$$$$
v_{2,0}(x, y, t) \frac{\partial v_{2,1}(x, y, t)}{\partial y}=0
$$

$$
\begin{aligned}
& p^{3}: \frac{\partial v_{2,3}(x, y, t)}{\partial t}-v_{1,2}(x, y, t) \frac{\partial v_{2,0}(x, y, t)}{\partial x}- \\
& v_{2,0}(x, y, t) \frac{\partial v_{2,2}(x, y, t)}{\partial y}- \\
& v_{1,0}(x, y, t) \frac{\partial v_{2,2}(x, y, t)}{\partial x}- \\
& v_{1,1}(x, y, t) \frac{\partial v_{2,1}(x, y, t)}{\partial x}- \\
& v_{2,1}(x, y, t) \frac{\partial v_{2,1}(x, y, t)}{\partial y}- \\
& v_{2,2}(x, y, t) \frac{\partial v_{2,0}(x, y, t)}{\partial y}=0 .
\end{aligned}
$$

Solving equations (28) through (35), and using the initial conditions (Eqs. (26)), one can find the fallowing results:

$$
\begin{aligned}
& v_{1,0}(x, y, t)=x^{2}, \\
& v_{1,1}(x, y, t)=2 x^{3} t, \\
& v_{1,2}(x, y, t)=5 x^{4} t^{2}, \\
& v_{1,3}(x, y, t)=14 x^{5} t^{3} .
\end{aligned}
$$

And

$v_{2,0}(x, y, t)=y$,

$v_{2,1}(x, y, t)=y t$,

$v_{2,2}(x, y, t)=y t^{2}$,

$v_{2,3}(x, y, t)=y t^{3}$.

According to the HPM, we can conclude that

$$
\begin{aligned}
& u(x, y, t)=\lim _{p \rightarrow 1} v_{1}(x, y, t)=\sum_{k=0}^{3} v_{1, k}(x, y, t), \\
& v(x, y, t)=\lim _{p \rightarrow 1} v_{2}(x, y, t)=\sum_{k=0}^{3} v_{2, k}(x, y, t) .
\end{aligned}
$$

After putting Eqs. (36) through (39) into Eq. (44) and Eqs. (40) through (43) into Eq. (45) the final results can be obtained as fallows: 


$$
\begin{aligned}
& u(x, y, t)=x^{2}+2 x^{3} t+5 x^{4} t^{2}+14 x^{5} t^{3}+\ldots \ldots \\
& v(x, y, t)=y+y t+y t^{2}+y t^{3}+\ldots \ldots
\end{aligned}
$$

\section{RESULTS AND DISSCUSION}

The final functional and numerical results are shown in table (1) and figures (1) Through (3). for equation (1) and equations. (3) respectively. In fact, in equation (1) the final results obtained from HPM were compared with the results of the exact solution, and in equation. (3) they compared with the results of the Adomian Decomposition Method ${ }^{[9]}$ which it is another approximate method for solving nonlinear partial differential equations. It is obvious that; considering more power of $p$ leads us to the more accurate results.

Table 1: Obtained results form the HPM in comparison to the ADM for Eq. 3.

\begin{tabular}{cc}
\hline HPM & $u(x, y, t)=x^{2}+2 x^{3} t+5 x^{4} t^{2}+14 x^{5} t^{3}+\ldots . .$, \\
HPM & $v(x, y, t)=y+y t+y t^{2}+y t^{3}+\ldots \ldots$, \\
ADM & $u(x, y, t)=x^{2}+2 x^{3} t+5 x^{4} t^{2}+14 x^{5} t^{3}+\ldots \ldots$, \\
ADM & $v(x, y, t)=y+y t+y t^{2}+y t^{3}+\ldots \ldots$ \\
\hline
\end{tabular}

\section{CONCLUSION}

In this letter, the homotopy perturbation method (HPM) was used for finding the approximate solutions of one-dimensional non-homogeneous parabolic partial differential equation with a variable coefficient and a system of nonlinear partial differential equations. In can be concluded that the HPM is very powerful and efficient technique in finding exact solutions for wide classes of problems. It is worth pointing out that the HPM presents a rapid convergence for the solutions.

The obtained solutions are compared with the exact solutions and the $\mathrm{ADM}^{[9]}$. the two solved examples show that the results of the present method are in excellent agreement with those obtained by the exact solution and ADM. The HPM has got many merits and much more advantages than the ADM. This method is to overcome the difficulties arising in calculation of Adomian Polynomials. Also the HPM does not require small parameters in the equation, so that the limitations of the traditional perturbation methods can be eliminated, and also the calculations in the HPM are simple and straightforward. The reliability of the method and the reduction in the size of computational domain gives this method a wider applicability. The results show that the HPM is a powerful mathematical tool for solving systems of nonlinear partial differential equations having wide applications in engineering.

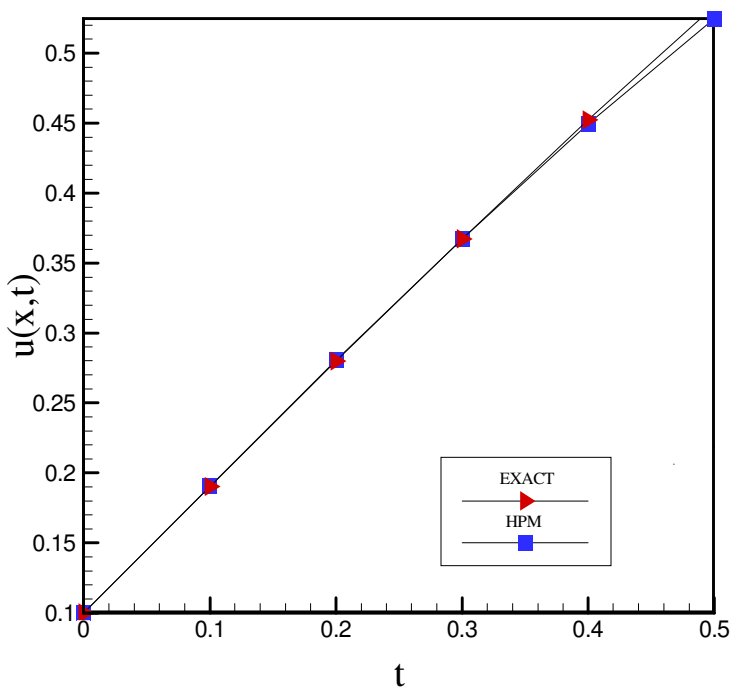

Fig. 1: Numerical compression of the results obtained by the HPM and the exact solution for Eq.1. $(x=0.1)$

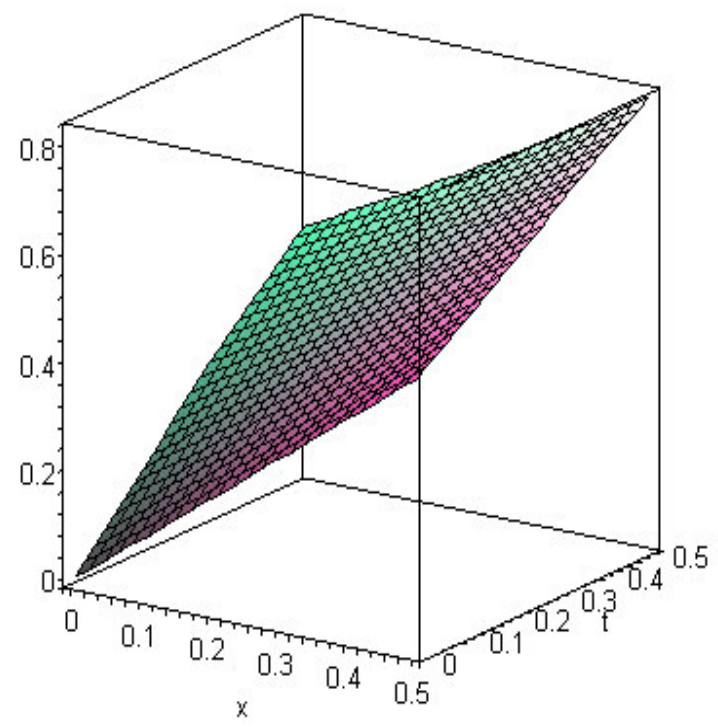

Fig. 2: The HPM result for $u(x, t)$ for Eq. $1(\mathrm{x}=0.1)$ 


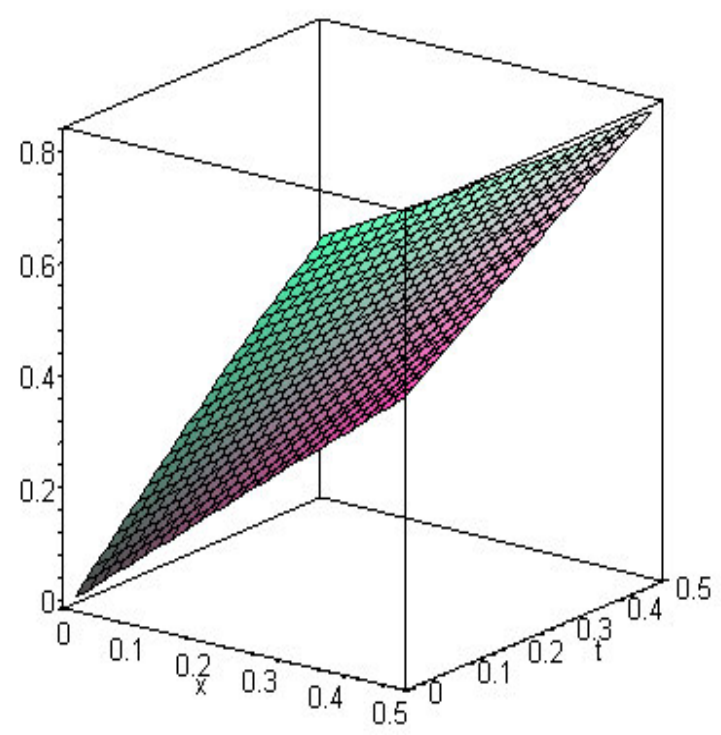

Fig. 3: Result of the exact solution for $u(x, t)$ for Eq.1 $(x=0.1)$

\section{REFRENCES}

1. He, J.H, 2005. Application of homotopy perturbation method to nonlinear wave equations. Appl. Math. Comput., 26 (3): 695-700.

2. He, J.H, 2006. Homotopy perturbation method for solving boundary value problems. Phys. Lett. A., 350 (1-2): 87-88.
3. He, J.H, 1999. Homotopy perturbation technique. Computer methods in applied mathematics and engineering., 178 (3-4): 257-262.

4. Aabbasbandy, S, 2006. Iterated He's homotopy perturbation method for quadratic Riccati differential equation. Appl. Math. Comput., 175 (1): 581-589.

5. Ganji, D.D., M.Rafei, 2006. Solitary wave solutions for generalized Hirota-Satusma coupled Kdv equation by homotopy perturbation method. Phys. Lett. A., 356 (2): 131-137.

6. He, J. H, 2000. A Coupling Method of A Homotopy Technique and A Perturbation Technique for Nonlinear Problems, International Journal of Nonlinear Mechanics, 35: 37-43.

7. He, J. H, 1997. A New Approach to Nonlinear Partial Differential Equations, Comm. Nonlinear Sci. Number. Simul. 2 (4) : 230-235.

8. Jang, B. 2007. Exact solutions to one dimensional non-homogeneous parabolic problems by the homogeneous Adomian decomposition method. . Appl. Math. Comput., 186: 969-979.

9. Gu, H., Z. Li, 2007. A modified Adomian method for system of nonlinear differential equations. Appl. Math. Comput., 187: 748-755. 